\title{
Selective detection of (E)-hexenal in the sub-ppm range and of ethanol in the low-ppm-range using semiconducting gas sensors with temperature cycled operation
}

\author{
André Kreiter ${ }^{1}$, Tilman Sauerwald ${ }^{1}$, Thorsten Conrad ${ }^{2}$, Andreas Schütze ${ }^{1}$ \\ ${ }^{1}$ Saarland University, Lab for Measurement Technology, Saarbruecken, Germany \\ e-mail: a.kreiter@Imt.uni-saarland.de \\ 2 3S GmbH, Mainzer Strasse 148, 66121Saarbruecken, Germany
}

\begin{abstract}
We use gas sensors with temperature cycled operation (TCO) for the detection of (E)-hexenal in the concentration range of 0.4 to $2.0 \mathrm{ppm}$ and ethanol in the concentration range 2.4 to $12 \mathrm{ppm}$. We are able to distinguish between these two reducing organic vapors in the ppm range and even to quantify (E)-hexenal under any ethanol presence in this range. This was achieved by calculating linear discriminant functions from the sensor reaction pattern to a stationary gas mixture flow. The obtained LDA-coefficients, subsequently applied to the same feature set obtained at another time, can classify the gas mixture with respect to these components in the mentioned concentration range. The temperature profile during cycling operation was selected due to our prior experience with the used sensor types.
\end{abstract}

Key words: Semiconducting gas sensors, temperature cycled operation, sub-ppm-sensitivity, organic vapors, linear discriminant analysis

\section{Introduction}

When plants suffer cellular damage, e.g. from insects [1] or microbes [2], they release gaseous substances, among them $(\mathrm{E})$-hexenal. These substances are the same for all plants since these events trigger the same lipoxygenase pathway. Monitoring the emissions thus can not only indicate health or damage of vegetables but also the quality of fruit [3]. The use of gas sensors for their detection instead of e.g. gas chromatography is a cost effective method opening wider application ranges, for example in food processing [4, 5]. Nevertheless, improvement is required since other organic vapors, e.g. ethanol as bacterial metabolite or component of cleaning agents in transport and storage, are also present in the application and interfere with the specific metaboliterelease driven sensor reaction. There are approaches in literature to distinguish between different organic vapors by using an array of multiple sensor elements [4, 6]. Nevertheless such a multisensor array is always increasing the expenses; furthermore drift effects and sensor instabilities are cumulating. Instead of a multisensory approach we use a virtual sensor array [7] based on temperature cycled operation (TCO). In this context, virtual means that only one sensing element, periodically operated at various temperature levels, is required instead of a sensing array with different materials and/or at different substrate temperatures. TCO can be used to increase selectivity by subsequent or online data processing, but also reduces sensor resistance drift during measurement [7]. We could show in a previous work, that even the detection of small concentrations (ethene at ppb levels) against a strong background (methane and carbon monoxide) are possible using a TCO virtual multisensor [8].

\section{Sensor selection}

We started setting up a virtual multisensor array by selecting an adequate sensor type. For this purpose, commercially available sensors have been compared with respect to their sensitivity characteristics. We also took into account the mechanical and electrical robustness; therefore, only ceramic plate type sensors were considered. Fig. 1 shows the results for $(E)$ hexenal exposure at $4 \mathrm{~V}$ heating voltage and $30 \%$ relative humidity (r.H.) for a sensor subset which was simultaneously operated under the same conditions. Finally, UST GGS 1330 T type [5], a robust $\mathrm{SnO}_{2}$ based thick film type sensor, seemed to be most suitable for this purpose. At $4 \mathrm{~V}$ heater voltage (corresponding to approx. $370^{\circ} \mathrm{C}$ sensor temperature), a sensor response of $29 \%$ increase of conductivity at 2 $\mathrm{ppm}$ and $34 \%$ at $3 \mathrm{ppm}$ (E)-hexenal was recorded; the nearest type, a GGS $5330 \mathrm{~T}$ with a response of $25 \%$ and $31 \%$, respectively, is from the same manufacturer. 


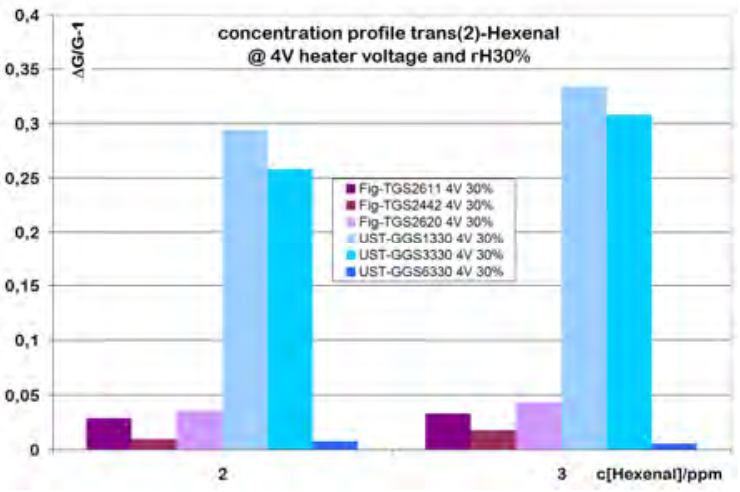

Fig. 1. Relative conductivity change at two low (E)hexenal concentrations for 6 sensor types at fixed heater voltage and constant humidity. "Fig" are sensor types from Figaro Inc, Japan; "UST" from Umweltsensortechnik GmbH, Germany.

\section{Sensor control and data acquisition}

In order to operate semiconducting gas sensor elements, a modular and versatile platform (3Stoolbox, [10]) was used. This platform was designed especially for (field) tests and supports a variety of commercially available semiconducting gas sensors. Via its graphical user interface different parametrizable temperature cycles and - depending on the combined set-up of the toolbox itself - application-specific flow cycles can be configured.

The core unit of the platform is a base-board with a powerful micro-controller communicating with external modules in a master-slave-configuration. The base-board is able to carry up to four gas sensor modules operating the actual sensing elements and additionally combined sensor/actor-modules for flow-cycle implementtation and ambient condition monitoring.

The platform outputs the sensor raw data (basically the measured voltages) which can easily be transformed into resistances or conductances. Via USB the platform is connected to a standard PC where the live data visualization and the storage is carried out.

Within the scope of the measurements in this paper, a platform with the following specification was used:

The temperature control allows a set-point accuracy of $2^{\circ} \mathrm{C}$ within an overall temperature range of 100 to $600{ }^{\circ} \mathrm{C}$. The set-point can be updated every $200 \mathrm{~ms}$.

The read-out circuit features a minimum sampling time of $1 \mathrm{~ms}$ with a measurement voltage accuracy of $5 \mathrm{mV}$ (using a 10-bit-ADC). The dynamic range of the read-out circuit can be adjusted according to the particular scenario under consideration.

\section{Temperature cycled operation (TCO)}

We parameterized a measurement system based on this 3S-toolbox [10] to repeat subsequent sensor temperature levels of $420^{\circ} \mathrm{C}$, $240^{\circ} \mathrm{C}$, and $330^{\circ} \mathrm{C}$ with $12 \mathrm{~s}$ duration each. These were similar to prior TCO experiments with this sensor type [11], although with a slightly reduced temperature cycle duration (36 $\mathrm{s}$ instead of $40 \mathrm{~s}$ ) with a data acquisition rate of $10 \mathrm{~Hz}$. The components of the data acquisition circuit for the sensor conductance measurement were chosen empirically to best match the conversion unit of the 3S-toolbox.

\section{Gas composition and exposition}

To obtain the sensor reaction data the sensors were placed in a gas-tight chamber and exposed to the programmed gas flow mixed from purified air (50\% r.h., $\mathrm{CO}_{2}$-free) with test gases (pressurized air/ethanol and $\mathrm{N}_{2} /(\mathrm{E})$ hexenal mixtures) using mass flow controllers (MFC). A constant flow rate of $500 \mathrm{sccm}$ was used during the measurements; the sensor was exposed to each gas mixture for $15 \mathrm{~min}$, followed by $45 \mathrm{~min}$ exposure to zero air.

\section{Results and discussion}

For the measurements in the scope of this paper, the above-mentioned platform has been equipped with two modules operating UST gas sensors of type GGS 1330. Thus, the sensors have been operated both statically, i.e. at constant temperature, as well as dynamically using TCO.

Fig. 2 shows the normalized dynamic sensor response $S$ to various concentrations of $(E)$ hexenal and ethanol resulting from the sensor raw conductance (digitized impedance converter readout). This normalized dynamic sensor response $S$ is calculated from the raw conductance $\mathrm{G}$ according to eq. 1 .

$$
S=\frac{G-G_{\min }}{G_{\max }-G_{\min }}
$$

We further calculated from $S$ secondary features, i.e. mean value $m v$, secant $s e c$, and length (of the time course of S) norm in each of four ranges of the temperature cycle (marked gray in fig. 2), resulting in a total of 12 features:

$$
\begin{aligned}
& m v=\frac{1}{i_{\text {End }}-i_{\text {Begin }}} \cdot \sum_{i=i_{\text {Begin }}}^{i_{\text {End }}} S_{i} \\
& \sec =\frac{S_{\text {End }}-S_{\text {Begin }}}{i_{\text {End }}-i_{\text {Begin }}}
\end{aligned}
$$




$$
\text { norm }=\sum_{i=i_{\text {Begin }}}^{i_{\text {End }}-1} \sqrt{1+\left(S_{i+1}-S_{i}\right)^{2}}
$$

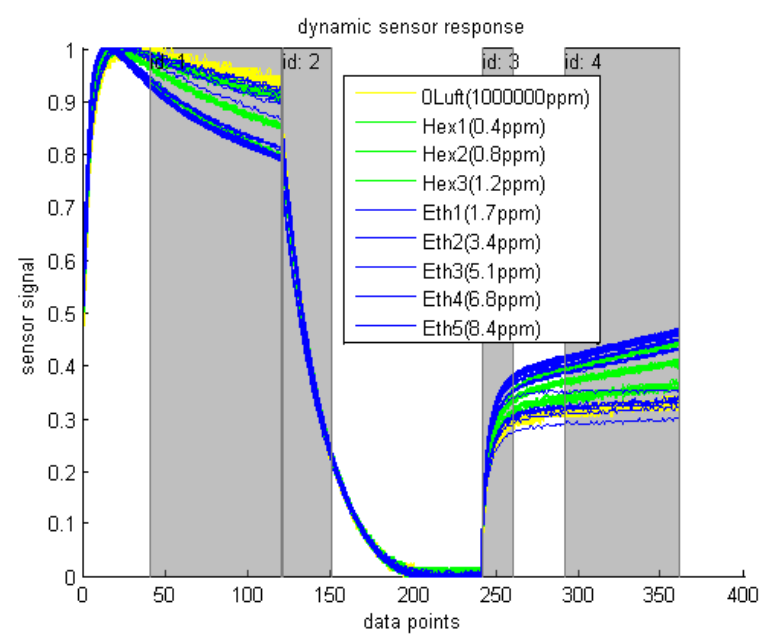

Fig. 2. Normalized dynamic sensor response. Data were acquired at a rate of $10 \mathrm{~Hz}$. Grey areas indicate the time intervals selected for feature extraction required for the first evaluation with $L D A$.

A data subset is used to perform a linear discriminant analysis (LDA) as a starting point. The subset is selected among those temperature cycles, which belong to phases of constant gas concentration and at least 2 repetitions of the same sensor resistance curve, in order to ensure proper working condition. For the LDA described here 32 different gas compositions (including zero air) were selected. We were able to show that the initially selected features are appropriate for separation of different concentrations of pure gases (fig. 3). Each circle or dot represents one temperature cycle data set projected into the dimensionally reduced space spanned by the discriminant function vectors formed as linear combinations of the mentioned features. Application of the obtained discriminant function coefficients to data obtained from further measurements using the same temperature cycle would allow identification of the gas composition, e.g. by using a nearest neighbor classifier.

Further improvement of resolution was achieved in two subsequent steps. First, redefining the ranges from which secondary features are calculated for LDA with respect to the geometric nature of the chosen parameters (fig. 4), increasing the number of features from 12 to 27 . Second, the $3^{\text {rd }}$ discriminant function calculated by the LDA was also taken into account. Both additions increase the performance considerably because all information contained in the cycle is taken into account and the feature space is exploited better.

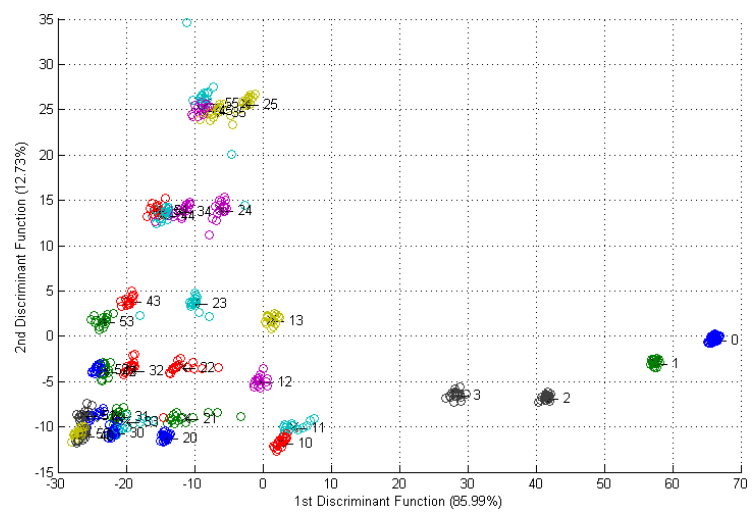

Fig. 3. LDA scatter plot (one circle represents one temperature cycle) discriminating pure (E)-hexenal and ethanol exposures as well as mixtures of both gases with a single UST GGS 1330 sensor. Legend: see Table 1.

\begin{tabular}{|c|c|c|c|c|c|c|c|}
\hline & \multicolumn{6}{|c|}{ Ethanol/ppm } \\
\hline & & 0 & 2.4 & 4.8 & 7.2 & 9.6 & 12 \\
\hline \multirow{6}{*}{ 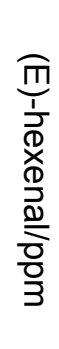 } & 0 & 0 & 1 & 2 & 3 & 4 & 5 \\
\hline & 0.4 & 10 & 11 & 12 & 13 & 14 & 15 \\
\hline & 0.8 & 20 & 21 & 22 & 23 & 24 & 25 \\
\hline & 1.2 & 30 & 31 & 32 & 33 & 34 & 35 \\
\hline & 1.6 & & & 42 & 43 & 44 & 45 \\
\hline & 2.0 & & & 52 & 53 & 54 & 55 \\
\hline
\end{tabular}

Tab. 1: Assignment of LDA scatter plot data to concentration combinations. The colored numbers refer to the dots' color in fig. 5 .

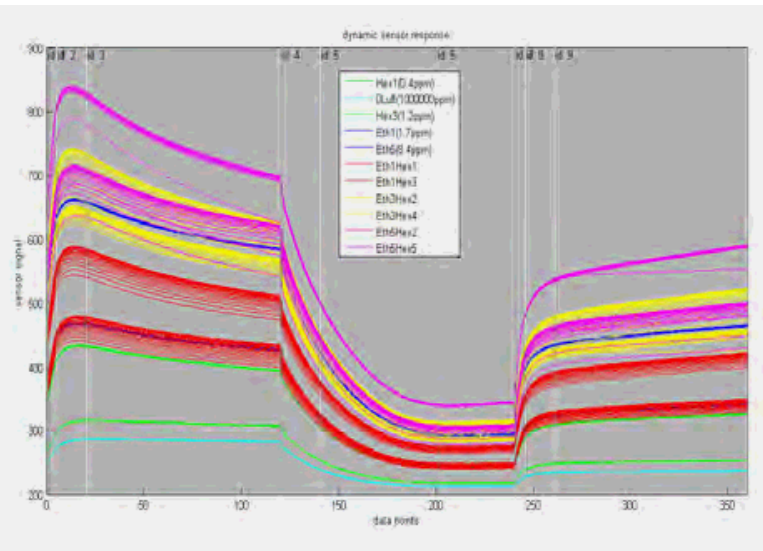

Fig. 4. Increasing and expanding time intervals, 3 within each temperature set point level for the extraction of 3 features each, resulted in a total of 27 secondary features per cycle.

For the first step we divided the 3 temperature cycle phases, i.e. temperature set points into 3 ranges each with respect to their different shapes: a narrow, rapid and steep change at the beginning, i.e. immediately after a temperature change; then, a section with pronounced curvature and, finally, the decay-like long end in 
order to obtain prominent contributions of the most suitable features. During this second adaption, it turned out that decreasing the number of features taken for the LDA stepwise did not visibly reduce the (improved) resolution. Thus, the overall result of these mathematical operations consisted of first increasing the number of time intervals and secondary features at the same time, but then reducing the number of features by selecting only those with the most significant contributions. In the end, the number of secondary features remains at the initial value thus keeping the numerical effort for the evaluation constant.

The 12 most significant features selected were the norm for intervals $2,3,5,6,8$, and 9 and the mean values for intervals $2,3,5,6,8$, and 9. The numbering scheme is "from left to right" in the time course of the cycles, i.e. mv2 means the mean value of the second time interval, ranging from .6 to $2.0 \mathrm{~s}$ within the $36 \mathrm{~s}$ cycle. Thus, 6 time intervals were suitable for increasing the selectivity; surprisingly, the secant as the most evident dynamic feature, does not provide significant input for the differentiation of the gases. Note that the number of cycles shown in fig. 4 has been greatly reduced for clarity showing only a subset of different gas mixtures. Also, fig. 4 shows the raw conductance data, not normalized values as in fig. 2 .

These improvements in LDA setup resulted in a clear distinction between several sub-ppm concentrations of (E)-hexenal even for varying concentrations of ethanol. Though regions without ethanol are separate, the different admixtures of ethanol do not lead to an overlap even for the sub-ppm ranges of (E)-hexenal as shown in fig. 5. This ability of the sensor together with the evaluation algorithm to quantify (E)-hexenal holds up even for 10 fold molar excess of ethanol.

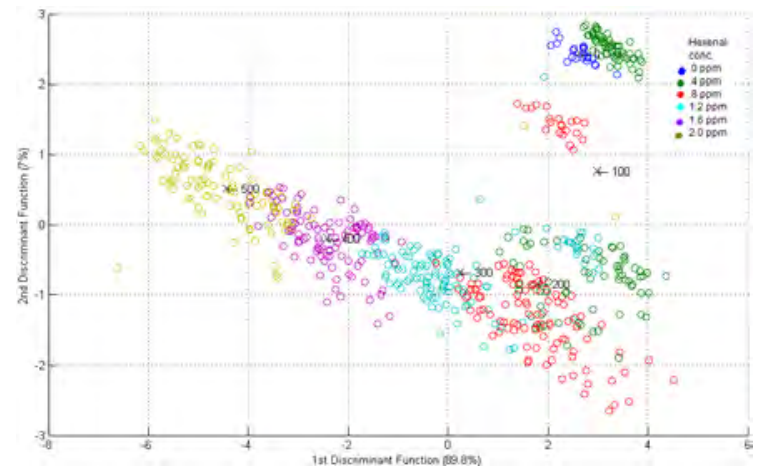

Fig. 5. Improved LDA scatter plot, showing the possibility to group hexenal concentration features even with differing ethanol concentrations. However, gas exposures with hexenal only (no ethanol background) are separated. Legend: see Table 1.
The increase in selectivity by employing the $3^{\text {rd }}$ LDA dimension can be estimated from the scree plot (fig. 6), where the bars for each dimension show their relative contribution. To depict these improvements, fig. 5 shows the projection of the discriminant function space for the first two dimensions. Although the clusters of data points for low concentrations of hexenal are not compact but actually divided into two compact regions (dark green circles representting 400 ppb hexenal).

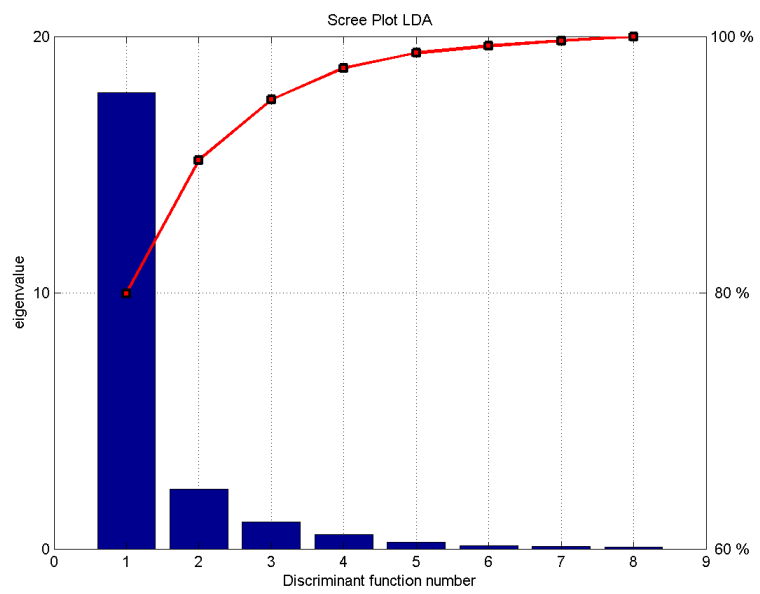

Fig. 6. Scree plot showing contributions of discriminant function vectors spanning the feature space

An estimate of the reliability of the processing comprises several parts. The one to be emphasized here is the stability of the positioning of TCO data points during a constant gas offer. We assume a minimum of 3 subsequent cycles under stationary gas flow required for valid feature extraction, but here we took at least 16 into calculation. The steadiness trajectories of the cycles' footprints, projected onto the axes of the space spanned by the discriminant functions obtained in this way, as they show a stable course, are used as indicators of this condition.

\section{Conclusion}

We could show that temperature cycled operation of single semiconductor gas sensors allows identification and quantification of low concentrations of (E)-hexenal in the sub-ppm range against a background of ethanol at much higher concentrations. The performance depends not only on the chosen temperature cycle but also on the evaluation algorithm with features extracted from the sensor raw data. A systematic approach to selecting suitable sensors, designing an optimal temperature cycle and an appropriate data evaluation allows efficient development of low cost gas sensor systems for various applications. 


\section{Acknowledgement}

This work was performed within a project funded by the Federal Ministry for Economics and Technology within the program ZIM.

\section{References}

[1] P. Paré, J. Thumlinson: "Plant volatile signals in response to Herbivore Feeding", Florida Entomologist (1995) 79(2), pp 93 - 103

[2] K.P.C. Croft, F. Jüttner, A.J. Slusarenko: "Volatile Products of the Lipoxygenase Pathway Evolved from Phaseolus vulgaris (L.) Leaves Inoculated with Pseudomonas syringae pv phaseolicola", Plant Physiol. (1993) 101, pp 13 - 24

[3] J. Vogt, F. Dunemann: "Molekularbiologische Charakterisierung der Lipoxygenase-Genfamilie des Apfels im Hinblick auf die Aromastoffproduktion in reifenden Früchten (Molecular characterization of the lipoxygenase gene family in apple (Malus domestica Borkh.) contributing to the production of flavour compounds in ripening fruits)“, Julius Kühn-Archiv (2011) Nr. 430, pp 20 $-28$

[4] P.-M. Schweizer-Berberich, S. Vaihinger, W. Göpel: "Characterisation of food freshness with sensor arrays", Sensors and Actuators B 18 (1994) pp 282 - 290
[5] J. Gardner, "A brief history of electronic noses", Sensors and Actuators B 18 (1994) pp 210 - 211

[6] Z. Xiaobo, Z. Jiewen: "Comparative analyses of apple aroma by a tin-oxide gas sensor array device and GC/MS", Food Chemistry Vol. 107 (2008), pp 120 - 128

[7] A. Schütze, A. Gramm, T. Rühl: "Identification of Organic Solvents by a Virtual Multisensor System with Hierarchical Classification", IEEE Sensors Journal Vol. 4, No. 6 (2004), pp 857 - 863

[8] P. Reimann, A. Schütze: "Fire detection in coal mines based on semiconductor gas sensors", Sensor Review 32/1 (2012), pp 47 - 56

[9] UST Umweltsensortechnik $\mathrm{GmbH}$, Geschwenda, Germany, http://www.umweltsensortechnik.de

[10] 3S Sensors, Signal Processing, Systems GmbH, Saarbruecken, Germany, http://www.3s-ing.de

[11] S. Horras: "Adaptierbares Messsystem zur Detektion von Gasen und Gerüchen basierend auf Halbleitergassensoren im temperaturzyklischen Betrieb“, Dissertation, Universität des Saarlandes (2011) 